\title{
59. Contribution to the Knowledge of Hydrogen Ion Concentration of the Lake Water in Japan.
}

\author{
By Shinkichi Yoshimura.
}

Geographical Institute, Faculty of Science, Tokyo Imperial University.

(Comm. by T. OKaDA, M.I.A., May 12, 1931.)

In recent years an enormous data on the hydrogen ion concentration of water of a large number of lakes in Japan ${ }^{11}$ have been accumulated and the knowledge of this chemical property of lake water has made a great progress.

Skadowsky recognized distinct types of lakes characterized chiefly by the amounts of buffer substances and by the production of phytoplankton. ${ }^{2}$ I have adopted the main idea of his opinion and classified the lake water into the following types:

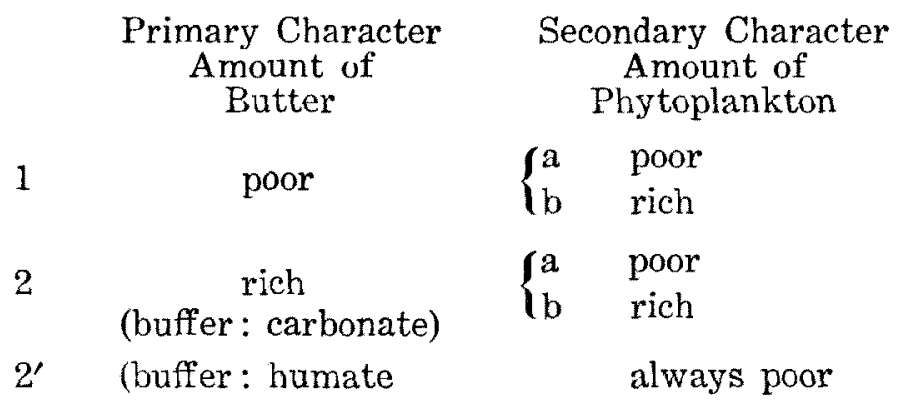

$\mathrm{pH}$ conditions of respective types are as follows:

\begin{tabular}{|c|c|c|c|c|c|}
\hline & \multirow{2}{*}{$\begin{array}{l}\text { Circulation } \\
\text { Period }\end{array}$} & \multicolumn{3}{|c|}{ Stagnation Period } & \multirow{2}{*}{$\begin{array}{l}\text { Annual } \\
\text { Range }\end{array}$} \\
\hline & & & per Layer & Lower Layer & \\
\hline 1 & $6.0-7.5$ & & $\begin{array}{l}6.0-7.5 \\
8.0-9.0\end{array}$ & $6.0-7.0$ & $\begin{array}{l}\text { small } \\
\text { biggest }\end{array}$ \\
\hline 2 & $7.0-8.5$ & & $\begin{array}{l}7.0-8.5 \\
8.0-9.0\end{array}$ & $7.0-8.0$ & $\begin{array}{l}\text { very small } \\
\text { big }\end{array}$ \\
\hline & always $4.0-6.0$ & & & & very small \\
\hline
\end{tabular}

1) All of the determination were made by the colorimetric method of Clarke.

2) Verhandl. Intern. Limnol. Kongr. Bd. 3 (1926), 109-144. 


\begin{tabular}{|c|c|c|c|c|c|c|c|}
\hline \multirow[b]{2}{*}{ Type } & \multirow[b]{2}{*}{ Name of Lake } & \multirow[b]{2}{*}{ Locality } & \multirow[b]{2}{*}{ Depth } & \multirow[b]{2}{*}{ Date } & \multicolumn{2}{|c|}{$\mathrm{pH}$} & \multirow[b]{2}{*}{ Observer } \\
\hline & & & & & $\begin{array}{l}\text { Sur- } \\
\text { face }\end{array}$ & $\begin{array}{l}\text { Bot- } \\
\text { tom }\end{array}$ & \\
\hline $1 a^{\prime}$ & $\begin{array}{l}\text { Ônuma-ike }{ }^{1)} \\
\text { Kutcharo-ko } \\
\text { Inawashiro-ko }\end{array}$ & $\begin{array}{l}\text { Nagano } \\
\text { Hokkaido } \\
\text { Fukushima }\end{array}$ & $\begin{array}{c}26 \mathrm{~m} \\
125 \\
96\end{array}$ & $\begin{array}{ll}\text { Aug. } & 1929 \\
\text { Aug. } & 1929 \\
\text { July } & 1930\end{array}$ & $\begin{array}{l}3.4 \\
5.1 \\
5.3\end{array}$ & $\begin{array}{l}3.4 \\
5.4 \\
5.5 \\
\end{array}$ & $\begin{array}{l}\text { A. Tanaka } \\
\text { K. Sawa } \\
\text { S. Yoshimura }\end{array}$ \\
\hline $1 \mathrm{a}$ & $\begin{array}{l}\text { Hakuba-ô-ike }{ }^{1)} \\
\text { Ontake } \\
\quad \text { San-no-ike }{ }^{1)}\end{array}$ & $\begin{array}{l}\text { Nagano } \\
\text { Nagano }\end{array}$ & $\begin{array}{l}13 \\
14\end{array}$ & $\begin{array}{l}\text { Oct. } 1927 \\
\text { Aug. } 1928\end{array}$ & $\begin{array}{l}5.3 \\
5.6\end{array}$ & $\begin{array}{l}5.3 \\
5.4\end{array}$ & $\begin{array}{l}\text { A. Tanaka } \\
\text { A. Tanaka }\end{array}$ \\
\hline & $\begin{array}{l}\text { Abashiri-ko } \\
\text { Akan-ko } \\
\text { Shikotsu-ko } \\
\text { Kutara-ko } \\
\text { Dôya-ko } \\
\text { Ô-numa } \\
\text { Tazawa-ko } \\
\text { Hibara-ko } \\
\text { Akimoto-ko } \\
\text { Chûzenji-ko } \\
\text { Ashi-no-ko } \\
\text { Motosu-ko } \\
\text { Kawaguchi-ko } \\
\text { Yamanaka-ko } \\
\text { Aoki-ko } \\
\text { Kizaki-ko } \\
\text { Nojiri-ko } \\
\text { Biwa-ko } \\
\text { Tane-ga-ike } \\
\text { Mi-ike } \\
\text { Ikeda-ko }\end{array}$ & $\begin{array}{l}\text { Hokkaido } \\
\text { Hokkaido } \\
\text { Hokkaido } \\
\text { Hokkaido } \\
\text { Hokkaido } \\
\text { Hokkaido } \\
\text { Akita } \\
\text { Fukushima } \\
\text { Fukushima } \\
\text { Tochigi } \\
\text { Kanazawa } \\
\text { Yamanashi } \\
\text { Yamanashi } \\
\text { Yamanashi } \\
\text { Nagano } \\
\text { Nagano } \\
\text { Nagano } \\
\text { Shiga } \\
\text { Tottori } \\
\text { Miyazaki } \\
\text { Kagoshima }\end{array}$ & $\begin{array}{r}17 \\
37 \\
363 \\
146 \\
183 \\
13 \\
425 \\
30 \\
32 \\
166 \\
43 \\
133 \\
15 \\
16 \\
58 \\
29 \\
38 \\
95 \\
13 \\
92 \\
233\end{array}$ & $\begin{array}{ll}\text { June } & 1927 \\
\text { July } & 1927 \\
\text { Aug. } 1928 \\
\text { Aug. } 1928 \\
\text { Aug. } 1928 \\
\text { July } 1928 \\
\text { Aug. } 1927 \\
\text { July } 1930 \\
\text { Aug. } 1930 \\
\text { Aug. } 1929 \\
\text { Sept. } 1930 \\
\text { July } 1929 \\
\text { July } 1929 \\
\text { July } 1929 \\
\text { Aug. } 1928 \\
\text { Aug. } 1928 \\
\text { July } 1928 \\
\text { Aug. } 1930 \\
\text { July } 1929 \\
\text { Aug. } 1929 \\
\text { Aug. } 1929\end{array}$ & $\begin{array}{l}7.3 \\
7.5 \\
7.7 \\
7.1 \\
7.2 \\
7.1 \\
6.7 \\
6.8 \\
6.6 \\
7.5 \\
6.8 \\
7.4 \\
7.6 \\
7.6 \\
7.1 \\
7.2 \\
7.3 \\
7.2 \\
6.7 \\
7.5 \\
7.3\end{array}$ & $\begin{array}{l}7.0 \\
7.0 \\
7.4 \\
7.1 \\
7.1 \\
6.9 \\
6.2 \\
6.0 \\
6.1 \\
7.1 \\
6.5 \\
7.4 \\
6.8 \\
6.8 \\
6.5 \\
6.5 \\
6.6 \\
6.8 \\
6.3 \\
6.5 \\
7.1 \\
\end{array}$ & $\begin{array}{l}\text { K. Sawa } \\
\text { K. Sawa } \\
\text { S. Yoshimura } \\
\text { S. Yoshimura } \\
\text { S. Yoshimura } \\
\text { S. Yoshimura } \\
\text { H. Tanakadate } \\
\text { S. Yoshimura } \\
\text { S. Yoshimura } \\
\text { D. Miyaji } \\
\text { S. Yoshimura } \\
\text { D. Miyaji } \\
\text { D. Miyaji } \\
\text { D. Miyaji } \\
\text { S. Yoshimura } \\
\text { S. Yoshimura } \\
\text { S. Yoshimura } \\
\text { S. Yoshimura } \\
\text { S. Yoshimura } \\
\text { S Yoshimura } \\
\text { S. Yoshimura }\end{array}$ \\
\hline $1 \mathrm{~b}$ & $\begin{array}{l}\text { Tôro-ko } \\
\text { Hangetsu-ko } \\
\text { Takasuka-numa } \\
\text { Suwa-ko } \\
\text { Koyama-ike } \\
\text { Sumiyoshi-ike } \\
\text { Unagi-ike } \\
\text { Towada-ko }\end{array}$ & $\begin{array}{l}\text { Hokkaido } \\
\text { Hokkaido } \\
\text { Saitama } \\
\text { Nagano } \\
\text { Tottori } \\
\text { Kagoshima } \\
\text { Kagoshima } \\
\text { Akita } \\
\end{array}$ & $\begin{array}{r}7 \\
18 \\
6 \\
7 \\
9 \\
26 \\
56 \\
325\end{array}$ & $\begin{array}{ll}\text { Sept. } 1928 \\
\text { July } 1928 \\
\text { Aug. } 1928 \\
\text { Aug. } 1927 \\
\text { July } 1929 \\
\text { Aug. } 1929 \\
\text { Aug. } 1929 \\
\text { Nov. } 1925\end{array}$ & $\begin{array}{l}8.8 \\
9.0 \\
8.7 \\
9.3 \\
8.8 \\
8.3 \\
8.0 \\
8.0\end{array}$ & $\begin{array}{l}6.8 \\
6.1 \\
7.0 \\
\\
7.3 \\
6.5 \\
6.8 \\
6.5\end{array}$ & $\begin{array}{l}\text { K. Sawa } \\
\text { S. Yoshimura } \\
\text { S. Yoshimura } \\
\text { A. Tanaka } \\
\text { S. Yoshimura } \\
\text { S. Yoshimura } \\
\text { S. Yoshimura } \\
\text { H. Tanakadate }\end{array}$ \\
\hline $2 a$ & $\begin{array}{l}(\text { L. Nipigon) } \\
\text { (Attersee) }^{3)}\end{array}$ & $\begin{array}{l}\text { Canada } \\
\text { Austria }\end{array}$ & $\begin{array}{l}123 \\
170\end{array}$ & $\begin{array}{ll}\text { Aug. } & 1923 \\
\text { Oct. } & 1924\end{array}$ & $\begin{array}{l}8.3 \\
8.3\end{array}$ & $\begin{array}{l}8.0 \\
7.8\end{array}$ & $\begin{array}{l}\text { W. A. Clemens } \\
\text { O. Haempel }\end{array}$ \\
\hline $2 \mathrm{~b}$ & $\begin{array}{l}\text { (L. Mendota) } \\
\text { (Victoria } \\
\text { Nyanza) }\end{array}$ & $\begin{array}{l}\text { Wisconsin } \\
\text { Africa }\end{array}$ & $\begin{array}{l}23 \\
80 \\
\end{array}$ & $\begin{array}{l}\text { Aug. } 1920 \\
\text { Sept. } 1927 \\
\end{array}$ & $\begin{array}{l}8.7 \\
8.5 \\
\end{array}$ & $\begin{array}{l}7.4 \\
7.5 \\
\end{array}$ & $\begin{array}{l}\text { C. Juday } \\
\text { M. Graham }\end{array}$ \\
\hline 2 & $\begin{array}{l}\text { Yashima-ike } \\
\text { Mizoro-ike }\end{array}$ & $\begin{array}{l}\text { Nagano } \\
\text { Kyoto }\end{array}$ & $\begin{array}{c}\text { shallow } \\
3\end{array}$ & Aug. 1928 & $\begin{array}{l}4.4 \\
4.1\end{array}$ & & $\begin{array}{l}\text { H. Nakano } \\
\text { S. Miki }\end{array}$ \\
\hline
\end{tabular}

(the shallow swamp or pond where water plants are growing are excluded from this table)

1) Pollutated by the inflowing water containing mineral acids from the volcanic region (subtype $1 a^{\prime}$ ).

2) Small pond in high mountain (about $2500 \mathrm{~m}$ ), extremely poor in electrolyte.

3) Univ. Toronto Studies. Biol. Ser. 25 (1924), 1-14.

4) Intern. Rev. d. ges. Hydrob. u. Hydrogr. 15 (1926).

5) Trans. Amer. Microscopical, Soc. (1924), 178-190.

6) Intern. Rev. d. ges. Hydrob. u. Hydrogr. 24 (1930), 328-357. 
It is concluded from the above observations that $\mathrm{pH}$ of the lake water in Japan possesses striking features as follows:

1. Nearly all of our lakes lie in volcanic region and they belong to soft water type. The alkalinity is usually less than 20-30 expressed in $\mathrm{mg} \mathrm{CaCO}_{3}$ per liter. No lake of hard water having a considerable size and depth has not yet been discovered.

2. A great number of soft water lakes have a poor production of phytoplankton (oligotrophic lake) ; so the vertical range of $\mathrm{pH}$ during the stagnation is small. Some of the deep volcanic lakes have no difference of it even at the depth 100 meters or more!

3. The rapid changes of $\mathrm{pH}$ in eutrophic lakes is due to the weak buffer of the water. The $\mathrm{pH}$ at the surface becomes very strongly basic and react sometimes 9.0 or more.

4. Some of the lakes in volcanic regions are pollutated by the water of the inflowing river in spite of their big capacity of water. Even Inawashiro-ko and Kutcharo-ko with surface extension of nearly $100 \mathrm{sqkm}$ and maximum depth of more than $100 \mathrm{~m}$, are entirely polluted by the inflowing acid water, and their production of phytoplankton is reduced by acidic reaction of water.

5. The lakes on high mountains have weak acidic reactions owing to the complete lack of buffer, for their contents of electrolyte are as poor as $5-10 \mathrm{mg}$ per liter. A single equilibrium of carbon dioxide is held between the air and the water. 